\title{
Treatment of irritable bowel syndrome with a novel colonic irrigation system: a pilot study
}

\author{
H.-H. Hsu ${ }^{1}$ - W.-H. Leung ${ }^{1}$ - G.-C. $\mathrm{Hu}^{2}$
}

Received: 30 August 2015 / Accepted: 7 March 2016/Published online: 19 May 2016

(c) The Author(s) 2016. This article is published with open access at Springerlink.com

\begin{abstract}
Background Medical treatments for irritable bowel syndrome (IBS) are often disappointing. A colonic irrigation system, the Ashong colonic irrigation apparatus (ACIA), was designed as a patient-administered device for defecation disorders. This pilot study evaluated the efficacy and safety of ACIA for IBS.

Methods Eighteen patients, 12 with constipation-dominant IBS (IBS-C) and 6 with diarrhea-dominant IBS (IBS-D) group, were studied. Patients were randomized into treatments of 1-4 weeks. Colonic irrigation was performed twice daily for 6 consecutive days per week. To determine the response to treatment, bowel movement frequency, stool consistency, abdominal pain, patient satisfaction with bowel movements, and distress/discomfort due to symptoms were assessed.

Results The scores of abdominal pain $(p<0.001)$, satisfaction $(p<0.001)$, and distress/discomfort $(p<0.001)$ improved significantly. The frequency of bowel movements in the IBS-C group increased from 1.68 to 3.78 times per week $(p<0.001)$. The occurrence of Bristol Stool Scale type 1 and 2 stool passage decreased from 45 to $13 \%$ $(p=0.009)$ in the IBS-C group and type 6 and 7 stools decreased from 62 to $28 \%(p=0.005)$ in the IBS-D group. Only mild adverse events occurred, and all patients completed treatment.
\end{abstract}

W.-H. Leung

leungwh22@gmail.com

1 Division of Colorectal Surgery, Department of Surgery, Mackay Memorial Hospital, 92, Sec. 2, Zhongshan N. Rd., Taipei City 10449, Taiwan

2 Department of Rehabilitation Medicine, Mackay Memorial Hospital, Taipei City, Taiwan
Conclusions Colonic irrigation with ACIA is safe and can improve abdominal pain, constipation, and diarrhea associated with IBS. Patients were more satisfied with their bowel movements and found their symptoms were less disturbing. Larger studies on long-term efficacy and quality of life and on placebo effects are needed.

Keywords Ashong colonic irrigation apparatus · Colonic irrigation · Irritable bowel syndrome

\section{Introduction}

Irritable bowel syndrome (IBS) is a functional gastrointestinal disorder according to the Rome III classification [1]. IBS affects $10-20 \%$ of the population, predominantly females. The pathogenesis of IBS involves abnormalities in motility, visceral sensation, brain-gut interaction, and psychosocial distress. More recently, altered gut immune activation, intestinal permeability, and the intestinal and colonic microbiome have been shown to contribute to IBS. Treatment of IBS includes psychological support, exercises, diet management, and medical treatments. The symptoms of constipation-dominant IBS (IBS-C) may be improved by fiber supplements, laxatives, and avoidance of short-chain carbohydrates and gluten. Anti-diarrheal medications, 5-HT3 antagonists, antispasmodics, and antidepressants are also effective [2]. A recent meta-analysis showed that administration of probiotics also improved symptoms and quality of life (QoL) for patients with IBS [3].

Rectal or colonic irrigation has been shown to be successful in treating neurogenic, congenital, and idiopathic bowel dysfunction; constipation; and fecal incontinence, as well as improving QoL [4-10]. Patients with fecal 
incontinence after low anterior resection may also benefit from colonic irrigation [11]. Medical treatments are often temporary and disappointing. The role of colonic irrigation for IBS, however, is not yet well established.

The Ashong colonic irrigation apparatus (ACIA) is a colonic irrigation system approved by the Taiwan Food and Drug Administration. It is a simple device designed for patient self-administration. The device is composed of a water tank, filter system, and an L-shaped disposable soft silicone rectal tube affixed to the toilet (Fig. 1). The water flow rate is maintained at $5 \mathrm{~mL}$ per second with a constant water temperature of $36 \pm 2{ }^{\circ} \mathrm{C}$. The duration of water flow can be adjusted as required. After sitting properly on the toilet and inserting the rectal tube, patient manipulates the ACIA via a remote control. The aim of this pilot study was to evaluate the efficacy and safety of AICA in the treatment of IBS.

\section{Materials and methods}

\section{Patient selection}

This clinical trial was approved by the Institutional Review Board of Mackay Memorial Hospital. Male and female IBS patients between 20 and 65 years of age who were refractory to previous medicinal treatments were invited for primary screening. Informed consent was obtained from all patients before the screening phase. Exclusion criteria included hernia, inflammatory bowel disease, grade 3 and 4 hemorrhoids, anal fissure, rectal prolapse, megacolon, peptic ulcer disease, previous abdominal surgery, renal impairment, uncontrolled hypertension, pregnancy, paralysis, and aortic aneurysm. All patients were assessed by double-contrast barium enema or colonoscopy to exclude any organic colonic lesions. Only those who met the Rome III diagnostic criteria of diarrhea-dominant IBS (IBS-D) and IBS-C [1] were included in the study.

\section{Treatment strategy}

ACIA was used with a constant water temperature of $37^{\circ} \mathrm{C}$, water flow at $5 \mathrm{~mL} / \mathrm{s}$ for $100 \mathrm{~s}$. Colonic irrigation was repeated for 8 cycles to constitute one complete therapeutic section. Patients were allowed to defecate at any time while irrigation was in progress.

Treatments were divided into 1-, 2-, 3- and 4-week courses for IBS-C (subgroups: 1C, 2C, 3C, 4C) and IBS-D (subgroups: 1D, 2D, 3D, 4D) groups in order to evaluate the influence of treatment duration on efficacy. Patients were randomized consecutively, beginning with the 4-week course and continuing through the 1 -week course, into each of the 8 treatment subgroups with the aim of producing 8 groups of 3 patients each. Patients received colonic irrigation twice daily for 6 consecutive days per week and were allowed to withdraw from the study at any time. A study nurse stayed beside the patients during irrigation, giving instructions, recording adverse effects, and
Fig. 1 a Overall view of the Ashong colonic irrigation apparatus. Tap water in the water tank flows through the filter system, then passes through the rubber tube, and finally reaches the rectal tube. b The rectal tube is fixed to the toilet. Filtered water flows out of the tip of the rectal tube in four directions, and the flow rate is maintained at $5 \mathrm{~mL} / \mathrm{s}$. c Diagram showing the method of rectal tube insertion
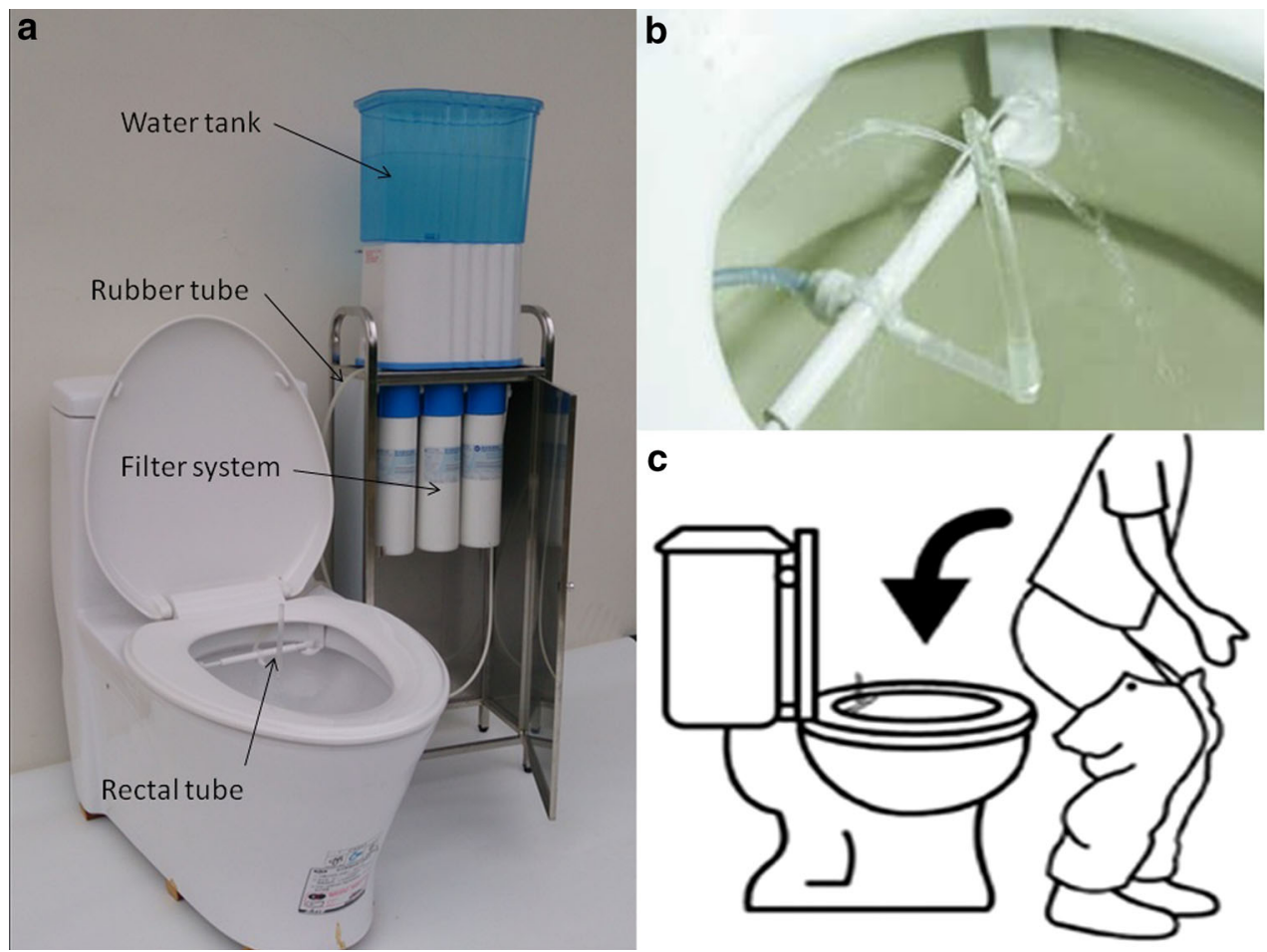
monitoring safety. After receiving a thorough explanation of the irrigation system, patients were instructed to manipulate the device themselves throughout the study period.

\section{Patient diary}

Patients maintained a record of bowel movement (BM) frequency, stool consistency, and abdominal pain intensity, from 7 days before treatment (pre-treatment phase) to 7 days following treatment (post-treatment phase). Stool consistency was recorded according to Bristol Stool Scale (BSS) (types 1-7) [12]. The scores of abdominal pain, satisfaction with $\mathrm{BM}$, and distress/discomfort due to symptoms were recorded on a 0 -to-10 rating scale. A score of 10 indicated the most severe abdominal pain, greatest satisfaction with $\mathrm{BM}$, and least distress/discomfort due to symptoms.

\section{Evaluation of efficacy and safety}

Efficacy was evaluated by measuring treatment-related changes in BM frequency, stool consistency, abdominal pain, satisfaction with BM, and distress/discomfort due to symptoms. All adverse events occurring in the course of the study period were recorded. A mild adverse event was defined as any mild symptom requiring neither medical intervention nor discontinuation of treatment. A moderate adverse event was defined as any intolerable symptom requiring medical intervention and terminating irrigation. A severe adverse event was defined as any life endangering event requiring immediate medical intervention. Patients were provided 24-h access to telephone assistance. Blood serum samples were obtained prior to treatment and 2 days ( \pm 1 day) before the end of treatment for analysis of glutamic-oxaloacetate transaminase (GOT), glutamic-pyruvic transaminase (GPT), gamma-glutamyl transpeptidase $(\gamma \mathrm{GT})$, alkaline phosphatase (ALKP), $\mathrm{Na}^{+}, \mathrm{K}^{+}, \mathrm{Cl}^{-}$, and blood urea nitrogen (BUN).

\section{Statistical analysis}

Demographic data and clinical characteristics were expressed as proportions for categorical variables and as mean \pm standard deviation (SD) values for continuous variables. The paired $t$ test or Wilcoxon signed-rank test was used to compare pre- and post-treatment changes in scores of abdominal pain, satisfaction with BM, and distress/discomfort due to symptoms. To assess the pre- and post-treatment changes in BM frequency and stool consistency, participants were enrolled in IBS-C and IBS-D subgroups. Differences between each of the variables were assessed using the two-tailed paired $t$ test or $\chi^{2}$ test, and values of $p<0.05$ were considered statistically significant. Analyses were carried out using SAS, version 9.0 (SAS, Cary, NC, USA).

\section{Results}

Between December 18, 2013, and July 18, 2014, 23 patients participated in the initial screening, and only 18 patients fulfilled the diagnostic criteria. One withdrew from the ongoing study, and 1 was excluded due to a diagnosis of cervical cancer. Two additional individuals were screened as potential substitutes for the missing 2 within the same study period, but unfortunately, only 6 patients were enrolled in the IBS-D group. Eighteen patients (13 females; mean age 45.2 (24-62) years) completed the treatments and entered the final analysis (Fig. 2). The IBS$\mathrm{C}$ group was female dominant, whereas the IBS-D group was male dominant (Table 1).

Overall abdominal pain score decreased significantly from 4.23 to $0.73(p<0.001)$ after colonic irrigation treatment. The score of satisfaction with $\mathrm{BM}$ increased from 3.10 to $6.52(p<0.001)$, and the score of distress/ discomfort due to symptoms improved from 2.95 to 6.75 $(p<0,001)$ (Fig. 3a). In addition, the results of the 2 individual groups showed significant improvements. After ACIA treatment, all patients in the IBS-D group and 5 of 12 patients in the IBS-C group experienced complete relief from abdominal pain.

BM frequency in the IBS-C group increased from 1.68 to 3.78 times per week $(p<0.001$; Fig. $3 b)$, and the percentage of BSS type 1 and 2 stool passage decreased from 45 to $13 \%$ ( $p=0.009$; Fig. $3 c)$ after treatment. Only 1 patient in the 1C subgroup showed no improvement in BM frequency, but still showed an improvement in stool consistency from BSS type 1 to type 4. Even though there was no significant improvement in BM frequency in the IBS-D group (Fig. 3b), the percentage of BSS type 6 and 7 stool passage decreased significantly from 62 to $28 \%$ $(p=0.005$; Fig. $3 c)$ after colonic irrigation. The only patient in the 1D subgroup showed persistent passage of loose stools, and this was the only patient from the IBS-D group showing no improvement in stool consistency.

One-third of patients experienced mild adverse events with neither discontinuation of treatment nor medical intervention. Abdominal cramping pain occurred in 2 patients $(11.1 \%), 4(22.2 \%)$ suffered from a sensation of mild abdominal fullness, 1 patient $(5.56 \%)$ experienced general weakness, and 1 presented with anal pain $(5.56 \%)$. Only 2 patients suffered from more than one adverse event.

Serum levels for the electrolytes $\mathrm{Na}^{+}, \mathrm{K}^{+}, \mathrm{Cl}^{-}$, before and after treatment, were all within normal ranges. Lower than normal limits of serum GPT, GOT, $\gamma \mathrm{GT}$, and BUN 


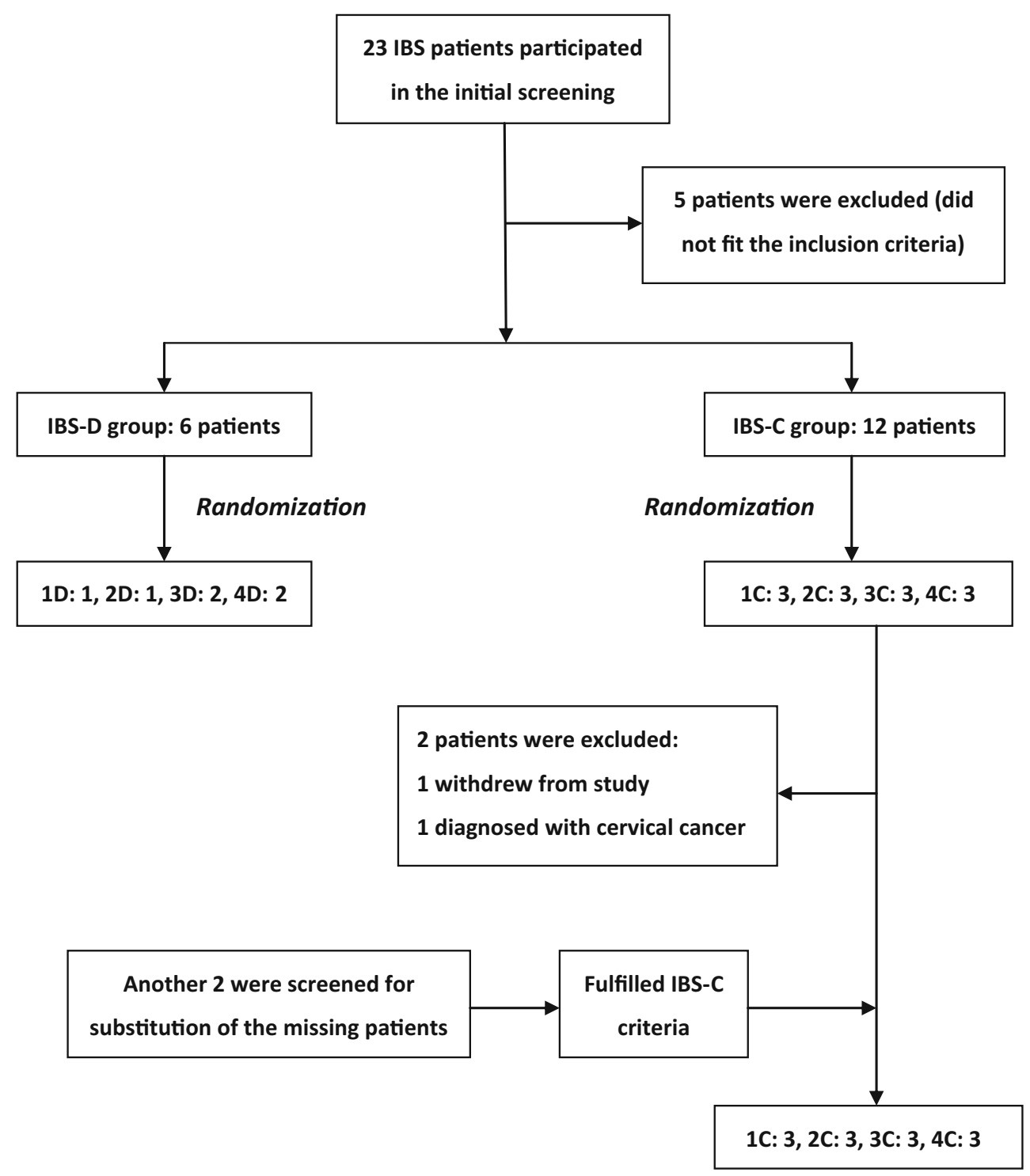

Fig. 2 Schematic flow chart from screening to randomization of participants

Table 1 Demographics of study participants

\begin{tabular}{llll}
\hline Group & All & IBS-C & IBS-D \\
\hline Sex & & & \\
$\quad$ Male & $5(28 \%)$ & $1(8.3 \%)$ & $4(67 \%)$ \\
$\quad$ Female & $13(72 \%)$ & $11(91.7 \%)$ & $2(33 \%)$ \\
Age (years) & & & \\
$\quad \begin{array}{l}\text { Mean } \pm \text { SD } \\
\text { Range }\end{array}$ & $45.2 \pm 13.6$ & $44.7 \pm 15.1$ & $46.0 \pm 11.2$ \\
\hline
\end{tabular}

$I B S$ - $C$ constipation-dominant irritable bowel syndrome, IBS-D diarrhea-dominant irritable bowel syndrome, $S D$ standard deviation

levels were observed before treatment in 8 participants $(44.4 \%)$. One male who was a heavy drinker had elevated basal and post-treatment levels of GOT and GPT.

\section{Discussion}

The beneficial effect of colonic or rectal irrigation on neurogenic or congenital defecation disorders is well known, and functional constipation and diarrhea have also been shown to benefit from rectal irrigation [13]. Even though the exact mechanism underlying the response of IBS to colonic irrigation is not known, our results demonstrate its potential benefit as an IBS treatment modality. A recent animal study by Zhang et al. [14] recognized methylglyoxal as an important cause of IBS symptoms. This bacterial product of anaerobic glycolysis in the large intestine elicits symptoms such as arrhythmia, headache, and diarrhea. Activation of NMDA receptors by methylglyoxol promotes visceral hypersensitivity, resulting 

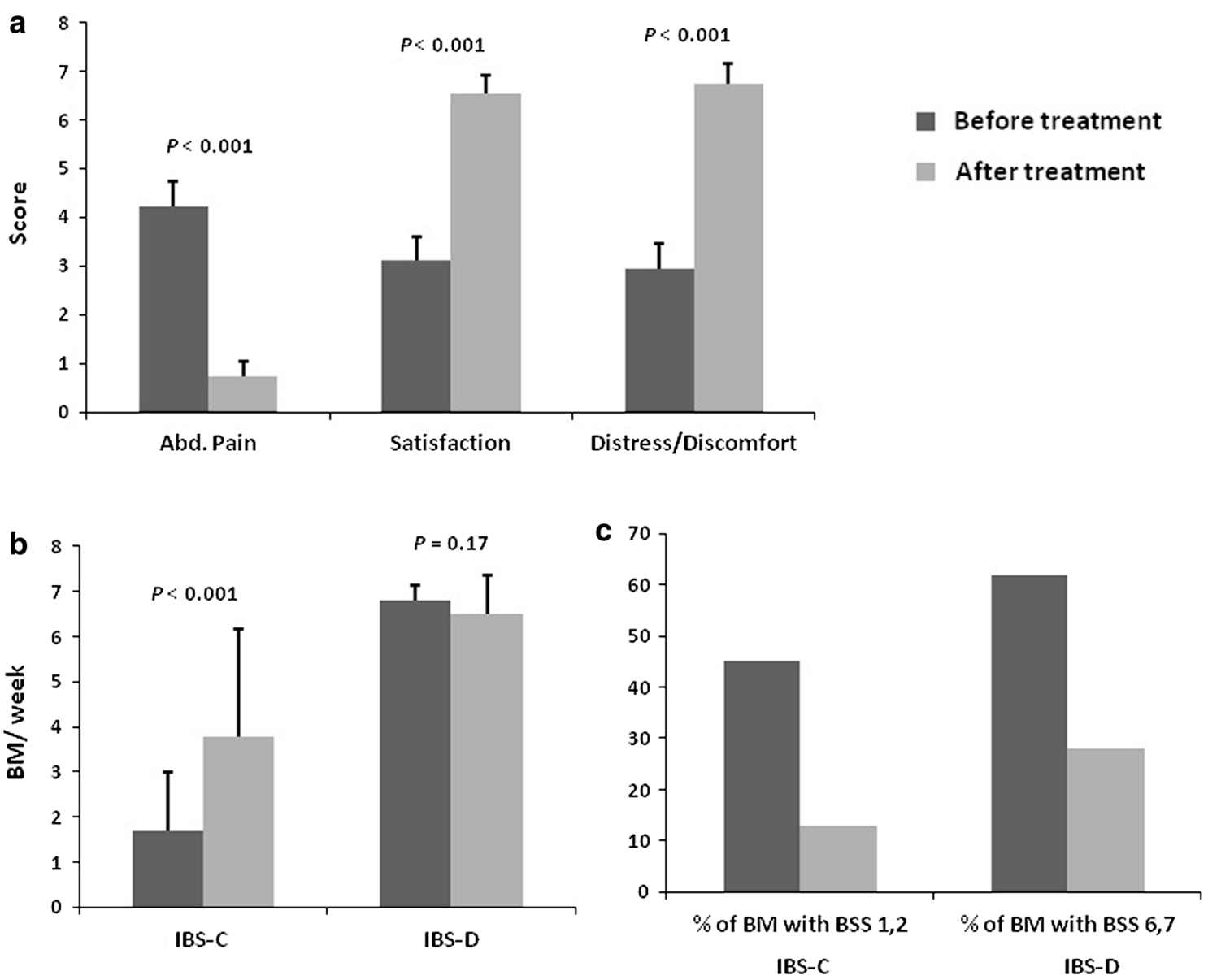

Fig. 3 a Scores of abdominal pain, satisfaction, and degree of distress/discomfort due to symptoms before and after treatment. Calculations involved all constipation-dominant irritable bowel

in increased 5HT secretion and peristalsis contributing to diarrhea. Visceral hypersensitivity reduces the threshold to noxious stimuli and bowel distension, providing a reasonable explanation for abdominal pain $[15,16]$, the major feature of IBS. We deduced that methylglyoxol and perhaps other metabolites could be washed out by water irrigation, and reverse visceral hypersensitivity. Zhang's study may serve to renew interest in the concept "body detoxification" by colonic irrigation, which is at present not well accepted by the medical professions [17].

Almost all participants with IBS-C experienced more than one additional BM per week than before treatment. Colonic irrigation also improved the stool consistency in most participants, whether this was for hard, lumpy stool in IBS-C or loose, watery stool in IBS-D patients. The results demonstrate colonic irrigation is effective in improving IBS-related constipation and diarrhea.

The influence of treatment duration on efficacy was investigated by dividing the treatment into 1 - to 4 -week courses. Abdominal pain, satisfaction with BM, and

syndrome and diarrhea-dominant irritable bowel syndrome patients. b Frequency of bowel movements before and after treatment. c Stool consistency changes in response to colonic irrigation

distress/discomfort due to symptoms were improved after initiation of colonic irrigation. This improvement was maintained throughout the treatment phase and even up to the post-treatment phase in every subgroup (data not shown). The treatment effects seemed to be independent of the treatment duration, but our short-term data are not adequate to support this conclusion.

Safety is always the greatest concern with bowel irrigation therapy, and rectal perforations and sepsis are not uncommon. Such complications have been attributed to malpractice by patients and non-medical personnel [1821]. However, the estimated rate of bowel perforation resulting from transanal irrigation is low, with a reported rate of $<0.002 \%$ [5, 7]. A well-devised treatment protocol used by experienced practitioners might reduce the risk of bowel perforation [20, 21]. Conversely, undiscovered conditions such as stercoral ulcer [19] or tumors could increase the risk of perforation. In this study, we provided complete colonic evaluations and detailed instructions for the treatment. Our patients experienced only mild adverse 
events such as sensation of abdominal fullness, weakness, and anal pain which did not necessitate either discontinuation of treatment or medical intervention.

A series of 36 cases of amebiasis cross-infection with 10 colectomies, in which 6 patients at a chiropractic clinic in Western Colorado died, was previously reported [22]. The outbreak led to the use of disposable, single-use parts of colonic irrigation devices [18], and patient-specific colonic irrigation system for defecation disorders are necessary to prevent cross-contamination.

Eight liters of water was required for a complete 1-day treatment, and water intoxication is an important safety concern in colonic irrigation. However, no neurological symptoms or altered serum biochemistry parameters, such as hyponatremia were observed in this study. In addition, since patients were allowed to defecate at any time during irrigation, the amount of water absorption via the colonic mucosa was very small. Lower than normal limit basal serum GOT, GPT, $\gamma \mathrm{GT}$, and BUN levels were observed in about $45 \%$ of the participants, suggesting these could be clinical signs of IBS. Moreover, colonic irrigation did not cause impairment in blood serum biochemistry or electrolyte imbalance. Chronic consumption of alcohol appeared to account for the only candidate with impaired basal and post-treatment GOT and GPT levels. Sacral nerve stimulation and the Malone antegrade continence enema procedure have been shown to be effective in patients with unremitting constipation and defecation disorders, improving QoL [23-26]. However, those are invasive procedures that come with a risk of surgical complications. Rectal or colonic irrigation is considered to be less invasive and effective as well [7, 8]. Irrigation treatment should be considered as the treatment of choice prior to surgical interventions $[4,13]$. The concept behind ACIA is no different from that behind the ordinary colonic irrigation sets [4, 7-9], but ACIA is simpler and more convenient.

Our results demonstrated that colonic irrigation with ACIA can be effective for IBS treatment. The improvement of abdominal pain, satisfaction with BM, and distress/discomfort due to symptoms indicated there was good relief of IBS-related problems and the patients were satisfied with the treatment. However, a placebo effect [27] should be taken into consideration. The positive effects on BM frequency and stool consistency are encouraging, and those are objectively measurable. QoL is always an important parameter for the effectiveness evaluation of IBS treatment. In this study, we focused on the feasibility of colonic irrigation on IBS treatment and QoL evaluation is therefore lacking.

The limitations of this study are the small sample size and short follow-up time.

\section{Conclusions}

The Ashong colonic irrigation apparatus is a simple, safe device that allows for patient self-administration of colonic irrigation. Our pilot study showed colonic irrigation can be effective in improving the abdominal pain, constipation, and diarrhea associated with IBS. However, the impact of placebo should be considered and is worth investigation. Larger studies with long-term follow-up are needed in order to draw more reliable conclusions.

\section{Compliance with ethical standards}

Conflict of interest The authors declare that they have no conflict of interest.

Ethical approval This study was approved by the committee of Institutional Review Board of Mackay Memorial Hospital, Taipei, Taiwan (MMH-IRB No. 13CT023b).

Informed consent Informed consent was obtained from all patients in this study.

Open Access This article is distributed under the terms of the Creative Commons Attribution 4.0 International License (http://crea tivecommons.org/licenses/by/4.0/), which permits unrestricted use, distribution, and reproduction in any medium, provided you give appropriate credit to the original author(s) and the source, provide a link to the Creative Commons license, and indicate if changes were made.

\section{References}

1. Longstreth GF, Thompson WG, Chey WD, Houghton LA, Mearin F, Spiller RC (2006) Functional bowel disorder. Gastroenterology 130:1480-1491

2. Chey WD, Kurlander J, Eswaran S (2015) Irritable bowel syndrome: a clinical review. JAMA 3:949-958

3. Didari T, Mozaffari S, Nikfar S, Abdollahi M (2015) Effectiveness of probiotics in irritable bowel syndrome: update systematic review with meta-analysis. World J Gastroenterol 21:3072-3084

4. Christensen P, Krogh K (2010) Transanal irrigation for disordered defecation: a systematic review. Scand J Gastroenterol 45:517-527

5. Faaborg PM, Christensen P, Kvitsau B, Buntzen S, Laurberg S, Krogh K (2009) Long-term outcome and safety of transanal colonic irrigation of neurogenic bowel dysfunction. Spinal Cord 47:545-549

6. Gardiner A, Marshall J, Duthie G (2004) Rectal irrigation for relief of functional bowel disorders. Nurs Stand 19:39-42

7. Christensen P, Krogh K, Buntzen S, Payandeh F, Laurberg S (2009) Long-term outcome and safety of transanal irrigation for constipation and fecal incontinence. Dis Colon Rectum 52:286-292

8. Corbett P, Denny A, Dick K, Malone PS, Griffin S, Stanton MP (2014) Peristeen integrated transanal irrigation system successfully treats faecal incontinence in children. J Pediatr Urol 10:219-222

9. Gosselink MP, Darby M, Zimmerman DD et al (2004) Long-term follow-up of retrograde colonic irrigation for defecation disturbances. Colorectal Dis 7:65-69 
10. Koch SM, Melenhorst J, van Gemert WG, Baeten CG (2008) Prospective study of colonic irrigation for the treatment of defaecation disorders. Br J Surg 95:1273-1279

11. Koch SM, Rietveld MP, Govaert B, van Gemert WG, Baeten CG (2009) Retrograde colonic irrigation for faecal incontinence after low anterior resection. Int J Colorectal Dis 24:1019-1022

12. Lewis SJ, Heaton KW (1997) Stool form scale as a useful guide to intestinal transit time. Scand J Gastroenterol 32:920-924

13. Chan DS, Saklani A, Shah PR, Lewis M, Haray PN (2012) Rectal irrigation: a useful tool in the armamemtarium for functional bowel disorders. Colorectal Dis 14:748-752

14. Zhang S, Jiao T, Chen Y, Gao N, Zhang L, Jiang M (2014) Methylglyoxal induces systemic symptoms of irritable bowel syndrome. PLoS ONE 26:e105307

15. Bouin M, Plourde V, Boivin M et al (2002) Rectal distension testing in patients with irritable syndrome: sensitivity, specificity, and predictive values of pain sensory thresholds. Gastroenterology 122:1771-1777

16. Kanazawa M, Hongo M, Fukudo S (2011) Visceral hypersensitivity in irritable syndrome. J Gastroenterol Hepatol (Suppl) 3:119-121

17. Acosta RD, Cash BD (2009) Clinical effects of colonic cleansing for general health promotion: a systemic review. Am J Gastroenterol 104:2830-2836

18. Dore M, Gleeson T (2015) Escherichia coli septic shock following colonic hydrotherapy. Am J Med 128:e31

19. Lundy JB, Gadacz TR (2006) Massive fecal impaction presenting with megarectum and perforation of a stercoral ulcer at the rectosigmoid junction. South Med J 99:525-527
20. Niv G, Grinberg T, Dickman R, Wasserberg N, Niv Y (2013) Perforation and mortality after cleansing enema for acute constipation are not rare but are preventable. Int $\mathrm{J}$ Gen Med 6:323-328

21. Taffinder NJ, Tan E, Webb IG, McDonald PJ (2004) Retrograde commercial colonic hydrotherapy. Colorectal Dis 6:258-260

22. Istre GR, Kreiss K, Hopkins RS et al (1982) An outbreak of amebiasis spread by colonic irrigation at a chiropractic clinic. N Eng J Med 307:339-342

23. Lundby L, Krogh K, Buntzen S, Laurberg S (2008) Temporary sacral nerve stimulation for treatment of irritable bowel syndrome: a pilot study. Dis Colon Rectum 51:1074-1078

24. Sharma A, Liu B, Waudby P, Duthie GS (2011) Sacral neuromodulation for the management of severe constipation: development of a constipation treatment protocol. Int J Colorectal Dis 226:1583-1587

25. Poirier M, Abcarian H, Nelson R (2007) Malone antegrade continent enema: an alternative to re section in severe defecation disorders. Dis Colon Rectum 50:22-28

26. Har AF, Rescorla FJ, Croffie JM (2012) Quality of life in pediatric patients with unremitting constipation pre and post Malone antegrade continence enema (MACE) procedure. J Pediatr Surg 48:1733-1737

27. Petal SM, Stason WB, Legedza A et al (2005) The placebo effect in irritable bowel syndrome trials: a meta-analysis. Neurogastroenterol Motil 17:322-340 